Published in Biochemistry Funct Integr Genomics. 2019 Sep;19(5):729-742. doi: 10.1007/s10142019-00680-5

\title{
Cryptochrome deletion in p53 mutant mice enhances apoptotic and anti-tumorigenic responses to UV damage at the transcriptome level
}

Ayse Derya Cavga ${ }^{1 \#}$, Mehmet Tardu#, Tuba Korkmaz ${ }^{3}$, Ozlem Keskin ${ }^{1}$, Nuri Ozturk ${ }^{3}$, Attila Gursoy ${ }^{\star}$, Ibrahim Halil Kavakli ${ }^{1,5^{*}}$

\# These authors contributed equally to this work.

${ }^{1}$ Chemical and Biological Engineering, Koc University, Istanbul, Turkey

${ }^{2}$ Computational Science Engineering, Koc University, Istanbul, Turkey

${ }^{3}$ Molecular Biology and Genetics, Gebze Technical University, Kocaeli, Turkey

${ }^{4}$ Computer Engineering, Koc University, Istanbul, Turkey

${ }^{5}$ Molecular Biology and Genetics, Koc University, Istanbul, Turkey

*Corresponding Authors:

Ibrahim Halil Kavakli

Email: hkavakli@ku.edu.tr

Tel:+90 2123381708

Attila Gursoy

Email: agursoy@ku.edu.tr

Tel:+90 2123381720

Keywords:

p53, Cryptochrome, circadian clock, cancer, RNA-seq, transcriptome 


\section{ABSTRACT}

Previous studies have demonstrated that deletion of Cryptochrome (Cry) genes protects $p 53^{-/-}$mutant mice from early onset of cancer and extends their median lifespan by about 1.5 fold. Subsequent in vitro studies had revealed that deletion of Crys enhances apoptosis in response to UV damage through activation of $p 73$, and inactivation of GSK3 $\beta$. However, it was not known at the transcriptome-wide level how deletion of Crys delays the onset of cancer in $p 53^{-/-}$mutant mice. In this study, RNA-seq approach was taken to uncover the differentially expressed genes (DEGs) and pathways following UV-induced DNA damage in $p 53^{-/}$and $p 53^{-/}$ Cry ${ }^{1 /-}$ Cry $2^{-/-}$mouse skin fibroblasts. Gene Set Enrichment Analysis with the DEGs demonstrated enrichment in immune surveillance associated genes regulated by IFN- $\mathrm{Y}$ and genes involved in TNFa signaling via NF-KB. Furthermore, protein network analysis enabled identification of DEGs $p 21$, Sirt1 and Jun as key players, along with their interacting partners. It was also observed that the DEGs contained a high ratio of non-coding transcripts. Collectively, the present study suggests new genes in NF-KB regulation and IFN-y response, as well as non-coding RNAs may contribute to delaying the onset of cancer in $p 53^{-1-C r y} 1^{1-C} \mathrm{Cry} 2^{-/-}$mice and increasing the life span of the these animals compared to $p 53^{-/}$mice. 


\section{INTRODUCTION}

The circadian clock regulates the physiology of organisms with a $\sim 24 \mathrm{~h}$ oscillatory period. It coordinates many physiological variables including oscillations in hormone secretion, blood pressure, and cognitive function (Takahashi et al. 2008). At the molecular level, the cellular clockwork involves both positive and negative transcriptional feedback loops. The BMAL1/CLOCK heterodimer is central to circadian biology, binding E-box elements (CACGTG) in the Period (Per) and Cryptochrome (Cry) genes and exerting a positive effect on their transcription in mammals (Kume et al. 1999; Panda et al. 2002). Then, the transcriptional repressors PER and CRY repress BMAL1-CLOCK activity and their own transcription (Gekakis et al. 1998; Vitaterna et al. 1999; Oishi et al. 2000; Shearman 2000; Kavakli et al. 2017).

Due to its widespread regulatory effects, it is expected that a relationship exists between the circadian clock and carcinogenesis, but the interplay is quite complex. Early research showed that arrhythmic mice with SCNlesions and Per2 mutant mice had faster tumor progression and higher rates of cancer incidence than control animals, respectively, which led to the generalization that disruption of the circadian clock would lead to increased propensity to cancer (Filipski et al. 2002; Fu et al. 2002). However, mice with deletions of Clock or Cry genes had similar rates of cancer incidence as wild type mice (Gauger and Sancar 2005; Antoch et al. 2008).

The subsequent case to examine was the effects of circadian alterations in a background of elevated propensity to cancer due to a mutation in a tumor suppressor or oncogene. The $p 53^{---}$mice were used as model organism to investigate such relationship between them since p53 is a key tumor suppressor protein that is known to be mutated in nearly half of all human cancers types (Attardi and Jacks 1999). The $p 53^{-/-}$mice were established to develop cancers such as lymphomas and lymphosarcomas, and therefore have a median lifespan of about 20 weeks, (Donehower et al. 1992; Attardi and Donehower 2005) which is quite dramatic since wild-type mice of the same strain have a mean life-span of about 2 years (Rowlatt et al. 1976).

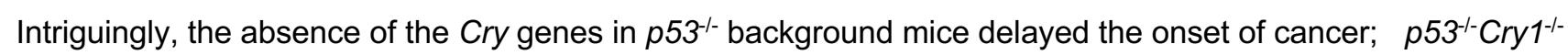
Cry $2^{-/-}$mice had about a 1.5 fold longer median lifespan compared to $p 53^{-/-}$mice (Ozturk et al. 2009). Several subsequent studies suggested that loss of Cry genes in $p 53$ mutants amplifies pathways of apoptosis in the absence of a functional $p 53$. The initial important observation was that $p 53^{-1-C r y} 1^{1-} \mathrm{Cry} 2^{-1-}$ mice skin fibroblasts (MSF) did not have reduced oncogenic transformation compared to $p 53^{-/}$mice, but were significantly more sensitive to UV-induced apoptosis (Ozturk et al. 2009). Detailed analysis of the augmented apoptotic behavior

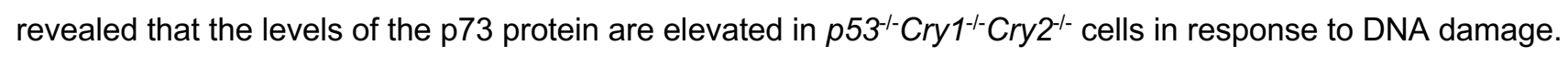
In response to oxaliplatin treatment, Cry mutants were observed to have elevated levels of EGR1, which was transactivating p73 (Lee and Sancar 2011a) and result in DNA-damage induced intrinsic apoptosis mechanisms. Notably, p73 is a member of the p53 protein family which has tumor suppressive functions that may become more apparent in a $p 53^{-/-}$knockout setting, and is known to regulate some of the genes in the p53 pathway (Fontemaggi et al. 2002; Harms et al. 2004; Lin et al. 2009).

Additionally, Cry mutation was also shown to promote extrinsic apoptosis via two mechanisms. Firstly, levels of pro-apoptotic cytokines such as TNFa were shown to be elevated in Cry mutant mice, causing an elevated inflammatory state (Hashiramoto et al. 2010). TNFa is a death ligand released by immune cells that induces 
apoptosis in the target cells where it binds its receptor, making it a key player in the antitumor response of the immune system (Wajant et al. 2003). Therefore, it is quite interesting that the levels of this cytokine are elevated upon loss of CRY protein, since this may be playing an important role in the extended lifespans of $p 53^{-1-C r y} 1^{-1-} \mathrm{Cry} 2^{-/-}$mice compared to $p 53^{-/-}$mice. A second and complementary extrinsic apoptosis mechanism observed after the deletion of Cry in $p 53^{-/-}$mutant mice involved the inactivation of GSK3 $\beta$, which fails to activate the anti-apoptotic activity of NF-KB, which causes cells to be more sensitive to the induction of apoptosis by TNFa (Lee and Sancar 2011b).

The present study aimed to elucidate transcriptome-wide changes in $p 53^{--} \mathrm{Cry}{ }^{1--} \mathrm{Cry} 2^{-1-}$ compared to $p 53^{---} \mathrm{MSF}$ cells in response to UV damage, to gain better insight about the delayed onset of cancer observed in the $p 53^{-1-}$

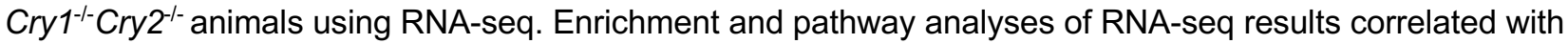
previous findings on the roles of TNFa signaling via NF-KB. In addition, these analyses revealed that additional genes downstream of IFNy response, as well as other important genes, including p21, Egr3, Casp1, Jun, Mmp9 and Sirt1 might contribute to the apoptosis of cancer cells in triple knockout animals. Transcriptomic analysis also revealed significant alterations in levels of novel long non-coding RNAs (IncRNAs) and pseudogenes, indicating their possible contribution to apoptotic response to UV damage in $p 53^{-1-} \mathrm{Cry} 1^{-1-} \mathrm{Cry} 2^{-1-}$ in MSF cells.

Collectively, present data suggests that a complex interaction between different pathways and non-coding RNAs may lead to enhanced apoptosis in $p 53^{-1-} \mathrm{Cry} 1^{-1-}$ Cry $2^{-/-}$animals.

\section{MATERIALS AND METHODS}

\section{Cell culturing and UV-treatment}

Mouse skin fibroblast (MSF) cells from $p 53^{-/-}$knockout and $p 53^{-1-} \mathrm{Cry} 1^{-1-} \mathrm{Cry} 2^{-/-}$mice in a C57BL/6J background were kindly provided from the laboratory of Aziz Sancar (University of North Carolina at Chapel Hill). Knockout MSF cells were maintained and grown in Dulbecco's Modified Eagle Medium (DMEM) supplemented with penicillin at $50 \mathrm{U} / \mathrm{ml}$, streptomycin at $50 \mu \mathrm{g} / \mathrm{ml}$, and 10\% fetal bovine serum. The cells were maintained in an incubator at $37^{\circ} \mathrm{C}$ under $5 \% \mathrm{CO}_{2}$.

For UV-treatment assays, cells were seeded in six-well plates at $4 \times 10^{5}$ cells per well and incubated in growth medium for 16-20 $\mathrm{h}$. Then, the cells were washed with 1x PBS (Phosphate Buffered Saline) and exposed to UV light (General electric germicidal lamp GT5, emitting mainly at $254 \mathrm{~nm}$ ) for 40 seconds, at a fluence of $40 \mathrm{~J}^{-2} \mathrm{~m}^{-2}$ with a fluence rate of $1 \mathrm{~J} \cdot \mathrm{m}^{-2} \cdot \mathrm{s}^{-1}$. UV light intensity was measured using a UVX Digital Radiometer (UVP, USA) at $264 \mathrm{~nm}$. After UV-treatment, the cells were washed with 1xPBS, growth medium was added, and cells were incubated for 16 hours. After 16 hours incubation, cells were harvested for RNA extraction followed by RNAseq library preparation. Non UV-treated cells were used as control samples. RNA-seq was performed using two biological replicates for each of the conditions shown in Fig. 1A.

\section{RNA extraction and RNA-seq library preparation}

Total RNA was extracted from $8 \times 10^{5}$ UV-treated and UV non-treated cells using RNeasy mini kit (Qiagen, USA) according to the manufacturer's instructions, and subjected to poly-A selection. The quality and quantity of RNA samples were assessed using Nanodrop 2000 spectrophotometer (Thermo Scientific, USA) and 2100 
BioAnalyzer (Agilent, USA). Only the RNA samples with OD260/280 between 1.8 and $2.2,28 \mathrm{~S}: 18 \mathrm{~S} \geq 1$ and the RNA integrity number (RIN) $\geq 8$ were used for library construction. After quality and quantity measurements, to remove residual genomic DNA, samples were treated with RNase-free DNase I (Thermo Scientific, USA) at a concentration of $1 \mathrm{U} / \mathrm{\mu g}$. Then, RNA-seq library preparation was performed using TruSeq mRNA Sample Preparation Kit (Illumina, USA) as described in previous work (Tardu et al. 2016; Sarayloo et al. 2017). Briefly, polyadenylated RNAs were purified from the $1 \mu \mathrm{g}$ of total RNA using oligo (dT) magnetic beads and fragmented using fragmentation buffer. After that, the cleaved short RNA fragments were used as a template for first-strand cDNA synthesis using first strand synthesis mix, and the second strand was synthesized using second strand marking master mix. AMPure XP beads (Beckman Coulter, USA) were used to clean up the double stranded cDNAs. Then, 3'end adenine nucleotides were added and sequencing adaptors were ligated to the cDNA fragments followed by enrichment of libraries by PCR amplification. Enriched cDNA libraries were used for cluster generation and sequencing. 100X2 paired-end sequencing of four CDNA libraries with two biological replicates were performed using the Illumina HiSeq 2500 sequencing platform (Illumina, USA).

\section{Transcriptome Analysis}

All sequence data were $2 \times 100 \mathrm{bp}$ in length. The high quality reads were saved in fastq files and deposited in the Gene Expression Omnibus (GEO) database at NCBI with the accession number GSE115102. The fluorescent images were processed into sequences, and base calling and quality value calculations were performed using the Illumina data processing pipeline (v1.5). The following steps were used for sequencing quality controls: (1) removing reads with adaptor contamination; and (2) removing low-quality bases (quality score < Q30) using Trimmomatic (v0.35) (Bolger et al. 2014). All downstream analyses were based on clean, high quality data.

STAR aligner (v2.5.2) (Dobin et al. 2013) was used to align reads to the mouse reference genome GRCm38. Following alignment, a Pearson's correlation analysis was performed to obtain the transcript-level $R^{2}$ value between replicates of each condition. After mapping, Cuffdiff command in Cufflinks suite (Hansen and lyengar) was used with default parameters to identify differentially expressed genes between UV-treated and nontreated cells. Quartile library normalization and pooled dispersion estimation methods were performed for the differential expression analysis. Fragments per kilobases of exons per million mapped read (FPKM) and fold changes were calculated for each sample.

Differential expression analysis was proceeded with transcripts with a minimum alignment count of 10 , a false discovery rate $(\mathrm{FDR})<0.01$, and an 'OK' status from Cufflinks from the $p 53^{-1-} \mathrm{Cry} 1^{-1-} \mathrm{Cry} 2^{-1-}$ and the $p 53^{-1-}$ nontreated vs. UV-treated differential expression analyses, which allowed them to be inter-compared. Among the mapped and differentially analyzed transcripts, an expression change was considered significant only if it was significant according the cutoff by Cufflinks, and if it showed a fold change more than $2\left(\log _{2} \mid\right.$ foldchange $\left.\mid \geq 1\right)$.

\section{Validation of RNA-seq results using qRT-PCR}

We randomly selected 11 representative down- and up-regulated differentially expressed genes for validation of RNA-seq using qRT-PCR under identical conditions where we performed RNA-seq experiments. Total RNA was extracted from $8 \times 10^{5}$ UV non-treated and UV-treated cells using RNeasy mini kit (Qiagen, USA) according to the manufacturer's instructions. After DNase I treatment, $1 \mu \mathrm{g}$ of total RNA was used for first-strand cDNA 
synthesis using random hexamer oligos. qRT-PCR was performed with Luminaris HiGreen qRT-PCR Master Mix (Thermo Scientific, USA) on the CFX Connect Real-Time PCR Detection System (Biorad, USA). qRT-PCR was performed with gene-specific primers (Supp. Table 1). Gapdh housekeeping gene whose expression level didn't change across UV(-) and UV(+) cells was used as an internal reference gene. The amplification programs were performed according to the following protocol: $95^{\circ} \mathrm{C}$ for $10 \mathrm{~min} ; 40$ cycles of $95^{\circ} \mathrm{C}$ for $2 \mathrm{~s}, 56^{\circ} \mathrm{C}$ for $10 \mathrm{~s}$ and $72{ }^{\circ} \mathrm{C}$ for $10 \mathrm{~s}$, and followed by a thermal denaturing step to generate the melting curves. All qRTPCR reactions were performed in biological triplicates (each triplicate with two technical replicates), and the results were expressed relative to the transcription level of Gapdh in each sample by using the 2- $\triangle \triangle C T$ method (Livak and Schmittgen 2001). The mRNA expression data analyzed by using IBM SPSS statistical 20.0 (SPSS Inc., USA). All data were presented as the means \pm S.D. $(n=6)$ of the relative mRNA expression.

\section{Identification of differentially expressed genes (DEGs)}

The focus of the present research was to identify the genes that were differentially expressed in response to UV treatment upon deletion of Cry genes in a $p 53^{-/-}$mutant background. Therefore, each gene was assigned a DEG Score according to the change in change they showed upon UV treatment in comparison of the $p 53^{-/-}$ Cry ${ }^{1--}$ Cry $2^{--}$cells to the $p 53^{--}$cells, as shown in Equation 1;

$$
\text { DEG Score }=\log 2\left(\frac{\left(\frac{\text { TKO_UV }}{\text { TKO_NT }}\right)}{\left(\frac{\text { PKO_UV }}{\text { PKO_NT }}\right)}\right)
$$

Equation 1

As described in Fig. 1B, significantly differentially expressed genes (DEGs) were identified as those with an absolute DEG Score above 1, indicating a change in change more than two fold. This threshold yielded 626 differentially expressed genes in response to UV-treatment in comparison of the $p 53^{-/-} \mathrm{Cry} 1^{-1-} \mathrm{Cry} 2^{-/-}$cells to the $p 53^{-1-}$ cells. As described in Fig. 1C, a DEG Score above 1 indicated an increased response to UV in $p 53^{-1-C r y 1-}$

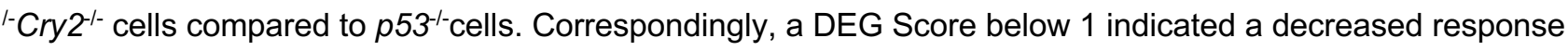

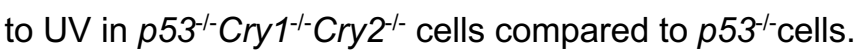

\section{Gene Set Enrichment Analysis (GSEA)}

The Gene Set Enrichment Analysis (GSEA) (Subramanian et al. 2005) was performed using the Differentially Expressed Gene Score (DEG Score, Eqn. 1) for each gene among the 626 DEGs as a pre-ranked input. Enriched gene sets were searched from the MSigDB Hallmark data collection using the default 'weighted' enrichment statistic parameter and 'meandiv' normalization, including gene sets with more than 15 and less than 5000 members. Enriched gene sets with a nominal $p$-value $<0.05$ were considered significant.

The MSigDB Hallmark gene sets are composed of genes that show consistent expression patterns in specific biological processes across many founder MSigDB gene sets based on publications. Since these gene sets have been carefully curated to include only genes that were consistently observed to be involved in a specific pathway, they have high reliability. The "HALLMARK_TNFA_SIGNALING_VIA_NFKB" set is made up of 200 genes regulated by NF-kB in response to TNF generated from 132 founder gene sets. The "HALLMARK_INTERFERON_GAMMA_RESPONSE" gene set is composed of 200 genes up-regulated in response to IFNG across 82 founder gene sets. The founder gene sets are obtained via expression profiling by array (Subramanian et al. 2005). 


\section{KEGG Pathway Enrichment Analysis}

KEGG Pathway enrichment was analyzed with the ClueGO Extension Tool of Cytoscape (Bindea et al. 2009). The 626 significant DEGs list was loaded as a Marker List and Mus musculus was selected. In the ClueGO Settings Section, the Pathway was selected as KEGG, and a threshold of significance was set as "Show only pathways with $\mathrm{pV} \leq 0.05$ ". The remaining settings were left as default. The resulting gene list in the "Functional Groups with Genes" output document was included in the Venn diagram.

\section{Protein-Protein Interaction Network Analysis}

Protein-protein interaction (PPI) networks can be constructed by extracting and visualizing protein-protein interaction data from databases, which can help determine key proteins within known networks. The degree of a node is defined as the number of edges linked to that node, therefore, in protein signaling networks, proteins with a very high degree number are interacting with many other proteins, which suggests a central regulatory role. Such proteins with high degree values are called "hub" proteins. (Tuncbag et al. 2009; Engin et al. 2012; Scardoni and Laudanna 2012; Cavga et al. 2015).

The betweenness centrality of a node is the number of times a node acts as a bridge along the shortest path between two other nodes. This reflects the amount of control that this node exerts over the interactions of other nodes in the network. In contrast to the measure of degree, the betweenness value favors nodes that join communities, rather than nodes that lie inside a community. Such proteins are called "bottleneck" proteins. Bottleneck proteins are thought to be biologically very significant, because even though they may not be making a lot of interactions, they make key interactions that play key roles in relaying a signal from one subnetwork to another (Freeman 1977; Yu et al. 2007).

In the present study, PPI network analysis in relation to the p53 protein was performed in order to further elaborate the biological mechanisms that may be involved in the delayed onset of cancer in $p 53^{-1-} \mathrm{Cry}{ }^{1-{ }^{-}} \mathrm{Cry} 2^{-{ }^{-}}$ compared to $p 53^{--}$knockout mice. STRING database was used to obtain experimentally validated PPI information (Jensen et al. 2009).

Of the $626 \mathrm{DEGs}$, ribosomal, proteasomal or polymerase-related genes were eliminated because since their interactions are the studied extensively, they dominate the network when they are included and disable the cancer related pathways from appearing. This elimination, and the elimination of genes unrecognized by STRING resulted in 487 genes to be used in the input list (Supp. Table 3).

The PPI network data was pulled from the STRING database (Jensen et al. 2009) with the following criteria; a maximum of 50 additional interactors per protein only in the first shell, based exclusively on experimental data and with a confidence score of 0.4 or above. The STRING output is provided in the references as a permanent link. (Jensen et al. 2018) The PPI network data obtained from STRING database was visualized and analyzed using Cytoscape (Shannon et al. 2003). The proteins were clustered with the default perfused force directed layout algorithm of Cytoscape and only slightly modified for better visualization. Detached nodes and pathways were deleted, leaving only the largest pathway that included p53 in focus. 


\section{RESULTS \& DISCUSSION}

\section{RNA-seq data analysis showed high unique mapping and coverage}

It has previously been shown that $p 53^{--} \mathrm{Cry} 1^{-{ }_{-1}} \mathrm{Cry} 2^{--} \mathrm{MSF}$ cells are more susceptible to ultraviolet (UV)-induced apoptosis than $p 53^{-/-}$MSF cells (Ozturk et al. 2009). To confirm this observation $p 53^{-/-}$and $p 53^{-{ }_{-1}} \mathrm{Cry} 1^{-I_{-}} \mathrm{Cry} 2^{-{ }_{-}}$ MSF cells treated with oxaliplatin as UV-mimetic agent are probed for apoptosis and searched for the presences of PARP cleavage, which is an indicator of the apoptosis. As expected, Cry knockout cells in the $p 53-$ null background ( $p 53^{-/}$, Cry $1^{-/}$, Cry $2^{-/-}$) had more apoptosis compared to only $p 53-$ null $\left(p 53^{-/}\right)$cells in response to oxaliplatin in agreement with previous findings of $p 53^{-1-} \mathrm{Cry} 1^{-1-} \mathrm{Cry} 2^{-1-}$ (Supp. Fig. 1) (Ozturk et al. 2009; Lee et al. 2013). Additionally, we also confirmed such phenomena are applicable in cancer cells, in the absence of Cry genes using the p53 mutant MDA-MB-231 breast cancer cell line. The results demonstrated that the absence of Crys resulted in enhanced PARP and Casp3 cleavage compared to $p 53$ mutant cells (Supp. Fig. 2 B,C) which is consistent with a previous study (Korkmaz et al. 2018). Additionally, survival rate of the MDA-MB-231 cells were determine for $24 \mathrm{~h}$ and $48 \mathrm{~h}$. They had comparable survival rate compared with wild type cells (Supp. Fig. 2D). After all these confirmations, we, then, aimed to identify genes and pathways that contribute to UV-induced apoptosis at the transcriptome level in these cell lines. The cells were exposed to UV and total RNA was isolated and sequenced as described in the Methods section (Fig. 1). After sequencing and de-multiplexing, RNA-seq data was aligned to the mouse reference genome GRCm38 using the STAR Aligner (Dobin et al. 2013).

Results indicated that the reads were uniquely mapped to gene loci (Fig. 2A). To evaluate reproducibility among biological replicates, Pearson's correlation tests were performed on the expression values. There were strong correlations between biological replicates for each condition (Pearson's correlation coefficients, $\left.R^{2}>0.95\right)$ (Fig. 2B).

All these quality control assessments allowed further analysis of the data to identify the differentially expressed genes (DEGs). For all analyses, only data from reads that uniquely mapped to the reference genome were used, and all multi-mappers were excluded. Differential expression analysis was performed using the Cuffdiff command in the Cufflinks suit (Trapnell et al. 2012) as described in Tardu et al (Tardu et al. 2016, 2017). The fold change in the number of mapped Fragments Per Kilobase of exon per Million mapped reads (FPKM) between UV-treated and non-treated samples were calculated using Cuffdiff.

Transcripts with a 0 (zero) FPKM read-out in any one of the 4 samples were eliminated due to causing division errors, however, the possible impact of including such transcripts in the analysis are discussed in below. These cutoffs yielded a total of 14659 differentially analyzable genes (Fig. 1B).

\section{Differentially Expressed Genes were confirmed via qRT-PCR}

The validity and reproducibility of the RNA-Seq data was confirmed via qRT-PCR analysis on 11 genes. Total RNA was isolated from both $p 53^{-/-}$and $p 53^{-1-} \mathrm{Cry} 1^{-1-} \mathrm{Cry} 2^{-1-}$ MSF cells were exposed to UV. After conversion of the total RNA into cDNA, real-time PCR (qRT-PCR) was performed using appropriate primers (Supp. Table 1). A comparison of qRT-PCR results and RNA-seq results revealed similar expression patterns for each gene, 
indicating that the RNA-seq and qPCR results were correlated, allowing confidence in the data and further analysis (Fig. 2C).

Identification of differentially expressed genes (DEGs) in $p 53^{-{ }^{-l}}$ Cry $1^{-I_{-}}$Cry $2^{-I_{-}}$cells compared to $p 53^{-I_{-}}$cells in response to UV

Analysis of the DEG results indicated that 5327 and 5112 genes were differentially regulated in response to UV

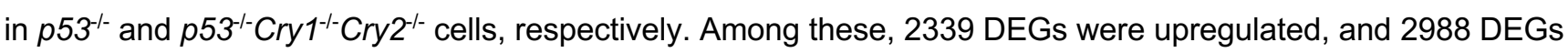
were downregulated in $p 53^{-/-}$cells while 2216 DEGs were upregulated and 2896 DEGs were downregulated in p53 $3^{--}$Cry $1^{-1-}$ Cry $2^{-/-}$cells (Fig. 1B, Fig. 2D).

Since the aim of this study was to identify the genes that were differentially expressed in response to UV treatment upon deletion of Cry genes in a $p 53^{-1-}$ mutant background, affected genes of interest were identified by comparing the change in FPKM value for each transcript in $p 53^{--} \mathrm{Cry} 1^{1--} \mathrm{Cry} 2^{-1-}$ UV-treated (TKO_UV) versus non-treated (TKO_NT) to the change in $p 53^{--}$UV-treated (PKO_UV) versus non-treated (PKO_NT). A gene was considered significant in terms of altered UV damage response in $p 53^{-1-} \mathrm{Cry}{ }^{1-{ }_{-1}} \mathrm{Cry} 2^{-{ }_{-}}$versus $p 53^{-/-}$cells only if it showed a change in change more than two folds; that is, if the absolute value of the DEG Score in Equation 1 was more than 1 (Fig. 1B).

This analysis resulted in a total of 626 transcripts. 267 of these transcripts were significantly differentially expressed in response to UV in both cell lines, and their fold changes were more than two-fold between the two cell lines. 189 genes were significantly differentially expressed in response to UV in $p 53^{-/-}$cells but not in $p 53^{-/-}$ Cry ${ }^{-{ }^{-}} \mathrm{Cry} 2^{-{ }^{-}}$cells, while 170 genes were significantly differentially expressed in response to UV in $p 53^{-1-C r y} 1^{-/-}$ Cry $2^{-/-}$cells but not in $p 53^{-/-}$cells (Fig. 1B). The DEG Score for each gene was calculated with Equation 1 , where genes that received a score above 1 were considered as "increased response" genes, and genes receiving a score below -1 were considered as "decreased response" genes (Fig. 1C, Fig. 2E). The up- or down-regulation statuses of these genes in response to UV within $\mathrm{p} 53^{-/-}$cells or $\mathrm{p} 53^{-1-} \mathrm{Cry} 1^{-{ }^{--}} \mathrm{Cry} 2^{-/-}$cells are also provided (Fig. 2F).

\section{Gene Set (GSEA) and KEGG Enrichment Analyses Identified Pathways that may Enhance Apoptosis in p53 ${ }^{-1-}$ Cry $1^{-1-}$ Cry ${ }^{-1-}$ MSF Cells upon Exposure to UV}

To functionally characterize the 626 DEGs and identify pathways, GSEA and KEGG Enrichment Analyses were performed (Subramanian et al. 2005). The GSEA results revealed that increased response genes were enriched in the Interferon-gamma (IFNY) response and tumor necrosis factor alpha (TNFa) signaling via nuclear factor kappa-B (NF-kB) related genes (Fig. 3A). Our results were consistent with a previous study, which had shown that the absence of the Cry genes sensitize $p 53^{- \text {- }}$ mutant cells to tumor necrosis factor $\alpha$ (TNF $\alpha$ )initiated apoptosis through NF-KB signaling, through the inactivation of GSK3 $\beta$ (Lee and Sancar 2011b). Another previous study also discussed that enhanced immune-surveillance of cancer cells may also help delay the onset (Ozturk et al. 2009). The present study showed that the increased response genes were enriched in genes upregulated during IFNy response, which is known to be essential for immunity against pathogens and for tumor surveillance (Schoenborn and Wilson 2007). Therefore, an increased IFNy response indicates an increased immune surveillance of tumor cells, which is likely to contribute to the delayed onset of cancer. 
GSEA also enabled identification of additional genes in these pathways (Fig. 3B) which possibly contribute to enhanced apoptosis in $p 53^{---}$mice upon deletion of Cry genes. Some of these genes act in IFNy response, such as Casp1, Casp4, and some act in TNFa signaling, such as Egr3 and Jun. Interestingly, some genes are involved in both pathways, such as Tnfaip3 and Cdkn1a. The KEGG pathway analysis allowed identification of genes such as Mmp9 and histones, which were enriched in the KEGG pathway "Transcriptional Misregulation in Cancer". We furthered confirmed the expression levels were changed in PKO and TKO cell lines with qPCR. Results indicated that the transcriptional level ere similar to those identified by RNa-seq (Supp. Fig. 3).

Tumor necrosis factor alpha-induced protein 3 (Tnfaip3, A20) is at the intersection of the IFNY response and

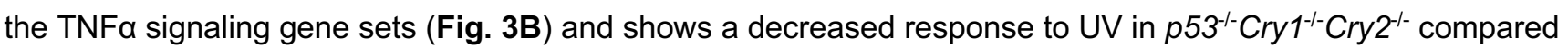
to $p 53^{-1-}$ knockouts (Supp. Fig. 3). This is particularly interesting because Tnfaip3 normally inhibits NF-kB activity and TNF-mediated programmed cell death (Ma and Malynn 2012) and decreased response can therefore contribute to increased apoptosis. Reduced Tnfaip3 response may also contribute to immune activation since Tnfaip3 is an important gatekeeper in immunity and Tnfaip3 deficient mice were observed to show severe inflammation (Lee et al. 2000; Coornaert et al. 2009).

Cyclin-Dependent Kinase Inhibitor 1A (Cdkn1a, p21) is also at the intersection of DEGs belonging to both the IFNY response and the TNFa signaling gene sets and it is among increased response genes (Supp. Fig. 4). $p 21$ is known to be a key apoptotic regulator in downstream of both p53 and p73 (Fontemaggi et al. 2002) and the $p 73$ tumor suppressor gene was previously shown to be specifically upregulated in the $p 53^{-1-C r y} 1^{1--} \mathrm{Cry} 2^{-1-}$ cells (Lee and Sancar 2011a). The cyclin-dependent kinase inhibitor CDKN1A binds to and inhibits the activity of cyclin-CDK2 or -CDK4 complexes, and therefore acts as a regulator of cell cycle progression at G1 (Wade Harper et al. 1993).

Another notable outcome of the GSEA is to identify up-regulation of Egr3 and Egr1 in the TNFa signaling gene

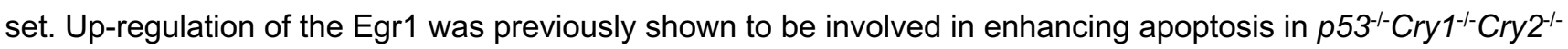
mice by transactivating p73 (Lee and Sancar 2011a). Our results suggest that the increased response in Egr3 may have a cooperative effect with Egr1 in this signaling pathway (Supp. Fig. 3).

Caspase-1 (Casp1) and Caspase-4 (Casp4) were observed to be among the genes that show an increased response to UV in $p 53^{--} \mathrm{Cry} 1^{-1-} \mathrm{Cry} 2^{-/-}$knockout cells compared to $p 53^{-/-}$knockout cells (Supp. Fig. 3). CASP1 is involved in the IFNy response which cleaves and activates the pro-inflammatory cytokines prolL-1 $\beta$ and prolL18. In fact, a CASP1-dependent programmed cell death named pyroptosis has recently been identified, and was observed to trigger an inflammatory response (Sollberger et al. 2014). Therefore, increased response of this gene may be triggering immune response in $p 53^{-1-} \mathrm{Cry} 1^{1-\mathrm{C}} \mathrm{Cry} 2^{-1-}$ knockout mice and contribute to delayed onset of cancer compared to $p 53^{-/-}$knockout mice.

$M y c$ is another particularly interesting DEGs identified in the TNFa signaling via NF-KB and transcriptional misregulation in cancer gene sets (Fig. 3B). Myc is a proto-oncogene that acts as a transcription factor in cell cycle progression, apoptosis and cellular transformation. Mutations, overexpression, rearrangement and translocation of $M y c$ have been associated various hematopoietic tumors, leukemias and lymphomas in humans (Hofmann et al. 2015). Interestingly, Myc is more downregulated (Supp. Fig. 3) by UV in the $p 53^{-1-}$ 


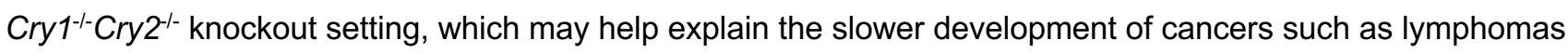
compared to the $p 53^{-1-}$ knockout mice.

KEGG pathway analysis showed enrichment in genes involved in transcriptional misregulation in cancer, and drew attention to a particular gene Mmp9, encodes matrix metalloproteinase 9 (Fig. 3B). Matrix metalloproteinases (MMPs) are involved in the breakdown of extracellular matrix, which makes them a suspect in cancer metastasis and angiogenesis. MMP9, is shown to be involved in breast cancer metastasis (van Kempen and Coussens 2016). Therefore, it may be important that Mmp9 has a decreased response to UV in the $p 53^{--} \mathrm{Cry} 1^{-1-} \mathrm{Cry} 2^{--}$MSF compared to the $p 53^{--}$MSF cells.

All these results suggested that additional genes are required in the important pathways that are likely to cause delayed onset of cancer upon Cryptochrome deletion in a $p 53^{---}$mutant background.

\section{Protein-protein interaction network (PPIN) analysis helps identify key protein interactions responsible for delayed onset of cancer}

In addition to the KEGG and the GSEA, PPIN analysis of the 626 DEGs was also performed to identify proteinprotein interactions that might play key roles related to cancer upon deletion of Cry genes in a p53 mutant background. Trp53 ( $p 53$ ) was included as a seed gene to reveal proteins that interact directly or indirectly with p53 when it is present, in order to understand which proteins might be interacting with p73 or similar proteins from this gene family that act when p53 is absent (Harms et al. 2004; Lin et al. 2009). The topological analysis of the PPI revealed the top hub and bottleneck proteins that are at key pathway intersections, as described in the methods section.

The PPI pathway analysis draws attention to Sirtuin 1 (SIRT1) as a protein that connects p53 related proteins to other key proteins in the network (Fig. 4), and it is among the significantly decreased response genes (Supp. Fig. 3). SIRT1 is a NAD-dependent protein deacetylase that can regulate the activity of key proteins involved in cancer (Luo et al. 2001). Most interestingly, Sirt1 mutant cells were shown to have increased apoptosis in response to stress, and SIRT1 was shown to interact with p73 and suppress its transcriptional activity (Luo et al. 2001; Jin et al. 2007). Therefore, it is particularly significant that the levels of this protein are decreased in $p 53^{-1-}$ Cry ${ }^{1--}$ Cry $2^{-1-}$ cells compared to $p 53^{-1-}$ cells (Supp. Fig. 3). This reduction in Sirt1 transcriptional levels may help explain the elevation of $p 73$ target genes such as $p 21$, and delayed onset of cancer due to increased apoptosis.

GSEA and PPI Network analyses suggested Jun as a key gene that was shown to have decreased response to UV damage in $p 53^{--} \mathrm{Cry} 1^{-1-} \mathrm{Cry} 2^{-1-}$ MSF cells compared to $p 53^{-1-}$ MSF cells. This observation is in agreement with the previous finding that Cryptochrome mutations sensitize $p 53^{---}$mutant cells to TNFa-induced apoptosis through NF-kB signaling (Lee and Sancar 2011b). Interestingly, c-Jun was previously shown to have a protective function from UV-induced cell death (Wisdom et al. 1999). The protection yielded by JUN may be lifted by Cryptochrome deletion, making the cells more prone to apoptosis.

\section{Correction of zero division errors allows gain of information on non-coding genes}


There is no consensus "best practice" on how to handle 0 (zero) FPKM readings during RNA-seq analysis; one published approach is to add 0.1 to the raw counts (Li et al. 2017), and another was to set a pseudo count of 0.001 (Rapaport et al. 2013) to all zero entries. Both approaches have been tried and evaluated. The first approach of adding 0.1 to the raw counts resulted in a reduced DEGs list of 487 genes. This approach resulted in loss of information because a large fraction of the differentially expressed genes have FPKM values between 0 and 1 , and an addition of 0.1 alters differential analyses. (Supp. Table 2)

The second approach of adding 0.001 to the raw counts resulted in a significant DEGs list of 696 genes, yielding 70 additional significant transcripts to the 626 that had been identified without changing the raw counts. (Supp. Table 4) This list of 696 genes was evaluated as an input gene list to the GSEA; this resulted in the same list of enriched Hallmark gene sets, but in this case, all of the gene sets were outside the significance threshold of a maximum nominal $P$ value of 0.05 . This was probably affected by the fact that the majority of the additional 70 genes have more significant DEG scores than the genes in the initial list since near-zero divisions produce large numbers, and therefore mask the significance ranking of other genes. (Supp. Table 4)

The MSigDB data collections of the GSEA tool are based on expression array data, which has much less sensitivity compared to RNA-seq data, and would not have been able to detect transcripts with near-zero expression levels. Since the gene set data of the GSEA were curated from expression arrays, it seemed unrealistic and unfitting to include the near-zero, sensitively obtained RNA-seq data in the GSEA analysis. Therefore, the GSEA analysis was carried out with the 626 DEGs that did not include the genes with zero FPKM values.

Importantly however, the majority of these 'zero cases' are non-coding transcripts, and some of these changes are at quite significant levels. For example, for the Gm26168 non-coding transcript in $p 53^{--} \mathrm{Cry} 1^{-1-} \mathrm{Cry} 2^{-1-}$ cells, the FPKM reading in the non-treated sample is 0 , while it moves up to an astonishing 706.18 FPKM reading upon UV treatment. (Supp. Table 4)

Of these additional 70 'zero case' genes, 46 are non-coding transcripts comprised of pseudogenes, long noncoding RNA (IncRNA) genes, or unclassified non-coding RNA genes according to the updated Mouse Genome Database, as shown in Fig. 5 (Supp. Table 4) (Smith et al. 2018).

Although the transcriptions of these non-coding RNAs were highly regulated by UV exposure in $p 53^{---C r y} 1^{-/-}$ Cry2 $2^{-1}$ cells, only a few of them are annotated and this make hard to extrapolate their function in delaying the onset of cancer. Two long non-coding RNA genes that have been annotated are pluripotency associated transcript 5 (Platr5) and pluripotency associated transcript 17 (Platr17), which have DEG scores of -9.84 and 8.71, respectively (Supp. Table 4). Interestingly, using weighted gene coexpression network analysis, these transcripts have previously been identified as IncRNAs associated with the pluripotent state of ESCs, clustering tightly among known pluripotency factors such as Nanog (Bergmann et al. 2015). Whether these noncoding RNAs play roles in apoptosis depending on the absence of Cry gens in p53 mutant background are yet to be shown. 
Interestingly, the transcriptional level of a noncoding transcript, which is not annotated Gm26168a, sharply increased from 0 to $706.18 \mathrm{FPKM}$ reading upon UV treatment of $p 53^{-1-} \mathrm{Cry} 1^{-1-} \mathrm{Cry} 2^{-1-}$ cells (Supp. Table 4). Such increase for the Gm26168 in response to UV is not observed in $p 53^{-/-}$cells (Supp. Table 4). Our data suggests that such novel noncoding RNAs may play roles in apoptosis in a Cry dependent manner in the absence of p53.

The comprehensive roles of these non-coding transcripts in the context of circadian clock disruption and p53 mutation will be better understood as RNA-seq analyses become more widely used to detect alterations in the levels of these transcripts in similar contexts, and as more non-coding transcripts are functionally characterized.

\section{CONCLUSION}

The GSEA and PPI network analyses of the differentially expressed transcripts identified by RNA sequencing demonstrated that deletion of both Cry1 and Cry2 genes in the $p 53^{-/-}$background causes increases in antiapoptotic and anti-tumorigenic pathways such as TNFa via NF-KB and immune-surveillance through IFNY, in response to UV damage. These results are in agreement with previous findings that implicated involvement of $\mathrm{NF}-\mathrm{KB}$ regulated cell autonomous mechanisms, and draw attention to the roles of enhanced immune surveillance and the possible involvement of key genes such as p21, Egr3, Sirt1, Jun, Mmp9 and Casp1.

The results obtained allow the proposition of the pathway model described in Fig. 6 . This model represents how the newly identified genes may be involved in the delayed onset of cancer upon deletion of Cry genes in p53 mutant background in light of previous knowledge. According to the results obtained, CASP1 and CASP4 may work under IFNY during immune response (Sollberger et al. 2014). TNFAIP3 and JUN, working under TNFa, can contribute to the regulation of NF-KB and enhanced apoptosis (Wisdom et al. 1999; Ma and Malynn 2012). p21 is possibly involved in the increase of apoptosis mediated by $p 73$, which is consistent with a previous report (Lee and Sancar 2011a) and the decrease in SIRT1 may result in activation of p73 and

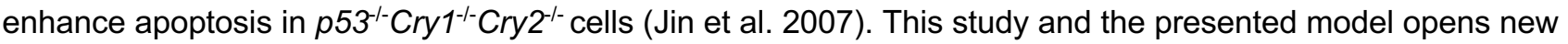
avenues for further investigation in explaining the delayed onset of cancer brought about by a Cryptochrome deletion in a p53 knockout background.

\section{ACKOWLEDGMENT}

This work was supported by grants from the Istanbul Development Agency grant ISTKA-TR/14/EVK/003 (to I.H.K.). This work was also partially supported by the Turkish Scientific and Technical Research Council (TUBITAK) grant 114S446 (to N.O.). A.G. and O.K. acknowledges the Science Academy, Turkey. 


\section{REFERENCES}

Antoch MP, Gorbacheva VY, Vykhovanets O, et al (2008) Disruption of the circadian clock due to the Clock mutation has discrete effects on aging and carcinogenesis. Cell Cycle 7:1197-1204. doi: 10.4161/cc.7.9.5886

Attardi LD, Donehower LA (2005) Probing p53 biological functions through the use of genetically engineered mouse models. Mutat Res Mol Mech Mutagen 576:4-21. doi: doi: 10.1007/s000180050269

Attardi LD, Jacks T (1999) The role of p53 in tumour suppression: Lessons from mouse models. Cell. Mol. Life Sci. 55:48-63

Bergmann JH, Li J, Eckersley-Maslin MA, et al (2015) Regulation of the ESC transcriptome by nuclear long noncoding RNAs. Genome Res 25:1336-1346. doi: 10.1101/gr.189027.114

Bindea G, Mlecnik B, Hackl H, et al (2009) ClueGO: A Cytoscape plug-in to decipher functionally grouped gene ontology and pathway annotation networks. Bioinformatics 25:1091-1093. doi: 10.1093/bioinformatics/btp101

Bolger AM, Lohse M, Usadel B (2014) Trimmomatic: a flexible trimmer for Illumina sequence data. Bioinformatics 30:2114-2120 doi: 10.1093/bioinformatics/btu170

Cavga AD, Karahan N, Keskin O, Gursoy A (2015) Taming Oncogenic Signaling at Protein Interfaces: Challenges and Opportunities. Curr Top Med Chem 1-25 doi: 10.2174/1568026615666150519101956

Coornaert B, Carpentier I, Beyaert R (2009) A20: Central gatekeeper in inflammation and immunity. J. Biol. Chem. 284:8217-8221 doi: 10.1074/jbc.R800032200

Dobin A, Davis CA, Schlesinger F, et al (2013) STAR: ultrafast universal RNA-seq aligner. Bioinformatics 29:15-21 DOI: 10.1093/bioinformatics/bts635

Donehower LA, Harvey M, Slagle BL, et al (1992) Mice deficient for p53 are developmentally normal but susceptible to spontaneous tumours. Nature 356:215-221. doi: 10.1038/356215a0

Engin HB, Keskin O, Nussinov R, Gursoy A (2012) A strategy based on protein-protein interface motifs may help in identifying drug off-targets. J Chem Inf Model 52:2273-2286. doi: 10.1021/ci300072q

Filipski E, King VM, Li X, et al (2002) Host circadian clock as a control point in tumor progression. J Natl Cancer Inst 94:690-697. doi: 10.1093/jnci/94.9.690

Fontemaggi G, Kela I, Amariglio N, et al (2002) Identification of direct p73 target genes combining DNA microarray and chromatin immunoprecipitation analyses. J Biol Chem 277:43359-43368. doi: 10.1074/jbc.M205573200

Freeman LC (1977) A Set of Measures of Centrality Based on Betweenness. Sociometry 40:35-41

Fu L, Pelicano H, Liu J, et al (2002) The circadian gene Period2 plays an important role in tumor suppression and DNA damage response in vivo. Cell 111:41-50. doi: 10.1016/S0092-8674(02)00961-3

Gauger MA, Sancar A (2005) Cryptochrome, circadian cycle, cell cycle checkpoints, and cancer. Cancer Res 65:6828-6834. doi: 10.1158/0008-5472.CAN-05-1119

Gekakis N, Staknis D, Nguyen HB, et al (1998) Role of the CLOCK Protein in the Mammalian Circadian 
Mechanism. Science (80- ) 280:1564-1569 \%U http://www.sciencemag.org/content/280. doi: 10.1126/science.280.5369.1564

Hansen J, lyengar R Analysis of RNASeq data using Tophat and Cufflinks to identify differentially expressed genes Analysis pipeline * Tophat Cuffdiff Differentially expressed

Harms K, Nozell S, Chen X (2004) The common and distinct target genes of the p53 family transcription factors. Cell Mol Life Sci 61:822-842. doi: 10.1007/s00018-003-3304-4

Hashiramoto A, Yamane T, Tsumiyama K, et al (2010) Mammalian Clock Gene Cryptochrome Regulates Arthritis via Proinflammatory Cytokine TNF- . J Immunol 184:1560-1565. doi: 10.4049/jimmunol.0903284

Hofmann JW, Zhao X, De Cecco M, et al (2015) Reduced Expression of MYC Increases Longevity and Enhances Healthspan. Cell 160:477-488. doi: http://dx.doi.org/10.1016/j.cell.2014.12.016

Jensen LJ, Kuhn M, Stark M, et al (2009) STRING 8-a global view on proteins and their functional interactions in 630 organisms. Nucleic Acids Res 37:D412-D416. doi: 10.1093/nar/gkn760

Jensen LJ, Kuhn M, Stark M, et al (2018) 487 items (mouse) - STRING interaction network. https://version-105.string-db.org/cgi/network.pl?networkId=CeX9QRITi9g5. Accessed 19 Oct 2018

Jin MD, Zhi YW, Dao CS, et al (2007) SIRT1 interacts with p73 and suppresses p73-dependent transcriptional activity. J Cell Physiol 210:161-166. doi: 10.1002/jcp.20831

Kavakli IH, Baris I, Tardu M, et al (2017) The Photolyase/Cryptochrome Family of Proteins as DNA Repair Enzymes and Transcriptional Repressors. Photochem. Photobiol. 93:93-103 doi: 10.1111/php.12669

Korkmaz T, Aygenli F, Emisoglu H, et al (2018) Opposite Carcinogenic Effects of Circadian Clock Gene BMAL1. Sci Rep 8:16023, doi:10.1038/s41598-018-34433-4

Kume K, Zylka MJ, Sriram S, et al (1999) mCRY1 and mCRY2 are essential components of the negative limb of the circadian clock feedback loop. Cell 98:193-205. doi: 10.1016/S0092-8674(00)81014-4

Lee EG, Boone DL, Chai S, et al (2000) Failure to Regulate TNF-Induced NF-kB and Cell Death Responses in A20-Deficient Mice. Science (80- ) 289:2350 LP-2354

Lee JH, Gaddameedhi S, Ozturk N, et al (2013) DNA damage-specific control of cell death by cryptochrome in p53-mutant ras-transformed cells. Cancer Res 73:785-791. doi: 10.1158/0008-5472.CAN-12-1994

Lee JH, Sancar A (2011a) Circadian clock disruption improves the efficacy of chemotherapy through p73mediated apoptosis. Proc Natl Acad Sci 108:10668-10672. doi: 10.1073/pnas.1106284108

Lee JH, Sancar A (2011b) Regulation of apoptosis by the circadian clock through NF-kB signaling. Proc Natl Acad Sci 108:12036-12041. doi: 10.1073/pnas.1108125108//DCSupplemental.www.pnas.org/cgi/doi/10.1073/pnas.1108125108

Li X, Brock GN, Rouchka EC, et al (2017) A comparison of per sample global scaling and per gene normalization methods for differential expression analysis of RNA-seq data. PLoS One 12:e0176185. doi: 10.1371/journal.pone.0176185

Lin YL, Sengupta S, Gurdziel K, et al (2009) p63 and p73 transcriptionally regulate genes involved in DNA repair. PLoS Genet 5:1-13. doi: 10.1371/journal.pgen.1000680 
Livak KJ, Schmittgen TD (2001) Analysis of Relative Gene Expression Data Using Real-Time Quantitative PCR and the 2- $\triangle \triangle C$ CT Method. Methods 25:402-408. doi: http://dx.doi.org/10.1006/meth.2001.1262

Luo J, Nikolaev AY, Imai S ichiro, et al (2001) Negative control of p53 by Sir2 $\alpha$ promotes cell survival under stress. Cell 107:137-148. doi: 10.1016/S0092-8674(01)00524-4

Ma A, Malynn B a (2012) A20: linking a complex regulator of ubiquitylation to immunity and human disease. Nat Rev Immunol 12:774-85. doi: 10.1038/nri3313

Oishi K, Fukui H, Ishida N (2000) Rhythmic Expression of BMAL1 mRNA Is Altered in Clock Mutant Mice: Differential Regulation in the Suprachiasmatic Nucleus and Peripheral Tissues. Biochem Biophys Res Commun 268:164-171. doi: 10.1006/bbrc.1999.2054

Ozturk N, Lee JH, Gaddameedhi S, Sancar A (2009) Loss of cryptochrome reduces cancer risk in p53 mutant mice. Proc Natl Acad Sci U S A 106:2841-2846. doi: 10.1073/pnas.0813028106

Panda S, Hogenesch JB, Kay SA (2002) Circadian rhythms from flies to human. Nature 417:329-335 DOI: 10.1038/417329a

Rapaport F, Khanin R, Liang Y, et al (2013) Comprehensive evaluation of differential gene expression analysis methods for RNA-seq data. Genome Biol 14:. doi: 10.1186/gb-2013-14-9-r95

Rowlatt C, Chesterman FC, Sheriff MU (1976) Lifespan, age changes and tumour incidence in an ageing C57BL mouse colony. Lab Anim 10:419-42. doi: 10.1258/002367776780956917

Sarayloo E, Tardu M, Unlu YS, et al (2017) Understanding lipid metabolism in high-lipid-producing Chlorella vulgaris mutants at the genome-wide level. Algal Res 28:244-252. doi: 10.1016/j.algal.2017.11.009

Scardoni G, Laudanna C (2012) Centralities Based Analysis of Complex Networks. New Front graph thory 323-348. doi: $10.5772 / 35846$

Schoenborn JR, Wilson CB (2007) Regulation of Interferon-y During Innate and Adaptive Immune Responses. In: Immunology BT-A in (ed). Academic Press, pp 41-101 DOI: 10.1016/S0065-2776(07)96002-2

Shannon P, Markiel A, Ozier O, et al (2003) Cytoscape: A Software Environment for Integrated Models of Biomolecular Interaction Networks. Genome Res 13:2498-2504. doi: 10.1101/gr.1239303

Shearman LP (2000) Interacting Molecular Loops in the Mammalian Circadian Clock. Science (80- ) 288:10131019. doi: $10.1126 /$ science.288.5468.1013

Smith CL, Blake JA, Kadin JA, et al (2018) Mouse Genome Database (MGD)-2018: Knowledgebase for the laboratory mouse. Nucleic Acids Res 46:D836-D842. doi: 10.1093/nar/gkx1006

Sollberger G, Strittmatter GE, Garstkiewicz M, et al (2014) Caspase-1: The inflammasome and beyond. Innate Immun 20:115-125. doi: 10.1177/1753425913484374

Subramanian A, Tamayo P, Mootha VK, et al (2005) Gene set enrichment analysis: A knowledge-based approach for interpreting genome-wide expression profiles. Proc Natl Acad Sci 102:15545-15550. doi: 10.1073/pnas.0506580102

Takahashi JS, Hong H-K, Ko CH, McDearmon EL (2008) The genetics of mammalian circadian order and disorder: implications for physiology and disease. Nat Rev Genet 9:764-775. doi: 10.1038/nrg2430 
Tardu M, Bulut S, Kavakli IH (2017) MerR and ChrR mediate blue light induced photo-oxidative stress response at the transcriptional level in Vibrio cholerae. Sci Rep 7:40817. doi: 10.1038/srep40817

Tardu M, Dikbas UM, Baris I, Kavakli IH (2016) RNA-seq analysis of the transcriptional response to blue and red light in the extremophilic red alga , Cyanidioschyzon merolae. Funct Integr Genomics 16:657-669. doi: 10.1007/s10142-016-0521-0

Trapnell C, Roberts A, Goff L, et al (2012) Differential gene and transcript expression analysis of RNA-seq experiments with TopHat and Cufflinks. Nat Protoc 7:562-78. doi: 10.1038/nprot.2012.016

Tuncbag N, Kar G, Gursoy A, et al (2009) Towards inferring time dimensionality in protein-protein interaction networks by integrating structures: the p53 example. Mol Biosyst 5:1770-8. doi: 10.1039/B905661K

van Kempen LCL, Coussens LM (2016) MMP9 potentiates pulmonary metastasis formation. Cancer Cell 2:251-252. doi: 10.1016/S1535-6108(02)00157-5

Vitaterna MH, Selby CP, Todo T, et al (1999) Differential regulation of mammalian Period genes and circadian rhythmicity by cryptochromes 1 and 2. Proc Natl Acad Sci 96:12114-12119. doi: 10.1073/pnas.96.21.12114

Wade Harper J, Adami GR, Wei N, et al (1993) The p21 Cdk-interacting protein Cip1 is a potent inhibitor of G1 cyclin-dependent kinases. Cell 75:805-816. doi: http://dx.doi.org/10.1016/0092-8674(93)90499-G

Wajant H, Pfizenmaier K, Scheurich P Tumor necrosis factor signaling. Cell Death Differ 10:45-65

Wisdom R, Johnson RS, Moore C (1999) c-Jun regulates cell cycle progression and apoptosis by distinct mechanisms. EMBO J 18:188-97. doi: 10.1093/emboj/18.1.188

Yu H, Kim PM, Sprecher E, et al (2007) The importance of bottlenecks in protein networks: correlation with gene essentiality and expression dynamics. PLoS Comput Biol 3:e59. doi: 10.1371/journal.pcbi.0030059 
Fig 1. Experimental setup and RNA-Seq analysis methodology. A) Mouse skin fibroblast (MSF) cells from $p 53^{-/}$(PKO) or $p 53^{--} \mathrm{Cry} 1^{-1-} \mathrm{Cry} 2^{-/}$(TKO) mice either received no treatment or were treated with UV, then total RNA was isolated and RNA sequencing analysis was performed as described in the flowchart. B) The genes of interest were selected as those with a significant TKO_UV vs. TKO_NT to PKO_UV vs. PKO_NT ratio above 2, as formulized. This resulted in 626 genes of interest that are differentially regulated in response to UV treatment in $p 53^{--} \mathrm{Cry} 1^{--} \mathrm{Cry} 2^{-/-}$cells compared to $p 53^{-/-}$cells. C) The significantly differentially expressed genes were scored and grouped as "increased response" genes and "decreased response genes" according to the formulized values.

Fig 2. RNA sequencing quality and content assessment. A) Mapping and coverage of RNA-seq reads. B) Expression comparison between 2 biological replicates for all 4 experimental groups. Correlation plots for transcript-level log10 $(\mathrm{FPKM}+1)$ values between replicates of $p 53^{--}$no treatment $(\mathrm{PKO} N \mathrm{NT}), p 53^{--} \mathrm{UV}$ treatment (PKO_UV), $p 53^{--C r y} 1^{-1-C r y} 2^{-/}$no treatment (TKO_NT) and $p 53^{--}$Cry $1^{-1-C r y} 2^{-/} \mathrm{UV}$ treatment(TKO_UV) cells. Pearson R2 values for the correlation are included as an insert in the plot. C) Validation of RNA-seq data with qRT-PCR. RNA-seq results of randomly selected 11 DEGs in log2 [(PKO_UV/PKO_NT)] and log2[(TKO_UV/TKO_NT)] were compared with qRT-PCR results. Blue bars represent relative transcript levels determined by qRT-PCR from three independent biological replicates $(n=6)$. Red bars represent transcript levels determined using RNA-seq. D) Up- and down-regulation status of the 14659 differentially analyzable genes, in $p 53^{--}$and $p 53^{--C r y} 1^{--C}$ Cry $2^{-/}$cells. A 'change' is considered as above 2 fold, and significant according to Cufflinks. Genes below these cutoffs are labeled 'no change'. E) The increased or decreased response status of the 626 selected significant DEGs in terms of their DEG Score= log2[(TKO_UV/TKO_NT)/(PKO_UV/PKO_NT)]. F) The up or down-regulation status of the 626 selected significant DEGs in terms of their fold changes as PKO_UV/PKO_NT and TKO_UV/TKO/NT

Fig 3. Gene set enrichment analysis and KEGG Pathway analysis of 626 DEGs demonstrate that Cryptochrome mutations in p53 mutant cells cause increased responses in IFNy response and TNFa signaling related genes, as well as transcriptional alterations significant in cancer. A) GSEA revealed that the 626 genes that were significantly differentiated in response to UV in $p 53^{--\mathrm{Cry}} 1^{-1-} \mathrm{Cry} 2^{-/-}$cells compared to $p 53^{--}$cells are enriched significantly in 2 MSigDB Hallmark gene sets. The HALLMARK_INTERFERON_GAMMA_RESPONSE gene set is composed of 200 genes up-regulated in response to IFNG across 82 founder gene sets. The HALLMARK_TNFA_SIGNALING_VIA_NFKB set is made up of 200 genes regulated by NF-kB in response to TNFa generated from 132 founder gene sets

(Subramanian et al. 2005). B) Venn diagram depicting genes from the 626 selected genes that belong to 2 MSigDB Hallmark gene sets and the KEGG Pathway "Transcriptional misregulation in cancer". KEGG Pathway enrichment was analyzed with the ClueGO Extension Tool of Cytoscape (Bindea et al. 2009).

Fig 4. Protein-protein interaction network analysis reveals key genes that interact with p53 associated genes. PPI network was constructed using STRING database for protein-protein interaction information, and Cytoscape for visualization and analysis (Shannon et al. 2003; Jensen et al. 2009). Interaction information is based exclusively on experiments and with a confidence score of 0.4 or above. A maximum of 50 additional interactors per protein were included. Of the $626 \mathrm{DEGs}, 487$ genes were used as seed genes as described in the Methods section in detail. The STRING output is provided in the references as a permanent link (Jensen et al. 2018). Ellipses represent nodes, which are proteins, and the lines between them represent protein-protein interactions, are called edges. The nodes are color coded according to their change in response to UV in $p 53^{-}$ ${ }^{1-C}$ Cry $1^{--} \mathrm{Cry} 2^{--}$MSFs compared to $p 53^{-/}$MSFs; green indicates increased response genes, red indicates decreased response genes. The blue nodes are additional nodes added by STRING database based on experimental interaction data with high confidence score. The node width represents the degree of each node, and the node height represents the betweenness centrality of each node; therefore, proteins with higher significance in the network appear bigger. The edge thickness and opaqueness increases with higher confidence score for the interaction from the STRING database. Top 10 seed genes with highest degrees and betweenness centralities are represented in the Venn diagram, and colored green if they are from the increased response genes, or red if they are from the decreased response genes. 
Fig 5. Elimination of transcripts with zero FPKM causes loss of information, especially on non-coding transcripts. A) The feature types of the 626 significantly differentially expressed genes (DEGs) in response to UV in $p 53^{--C r y} 1^{--} \mathrm{Cry} 2^{-/-}$cells compared to $p 53^{-/-}$cells. B) The feature types of 70 significant DEGs that were eliminated due to a lack of a consensus approach on how to handle 0 (zero) FPKM readings that cause division errors. 46 out of 70 such DEGs were non-coding transcripts, with examples such as 0 to 706.18 FPKM changes between TKO_NT to TKNO_UV, which are actually quite significant (Supp. Table 4). Feature type information obtained from the MGI database (Smith et al. 2018).

Fig 6. Proposed pathway model for involvement of novel candidate genes in the delayed onset of

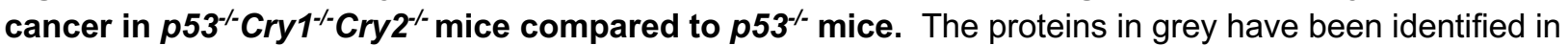
previous research to be involved in increased apoptotic levels after Cry deletions in $p 53^{-/-}$cells. The current RNA-seq analysis has drawn attention to the possible involvement of the genes in red and green, after UV

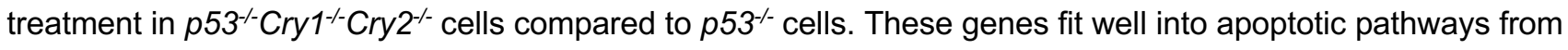
previous knowledge. Of the dark blue lines between proteins, arrow-headed lines indicate induction, while barheaded lines indicate inhibition. Undashed lines indicate findings from previous studies, dashed lines indicate interactions proposed by present analyses that are supported by known interactions from the literature (Fontemaggi et al. 2002; Jin et al. 2007; Lin et al. 2009; Ozturk et al. 2009; Hashiramoto et al. 2010; Lee and Sancar 2011a). 


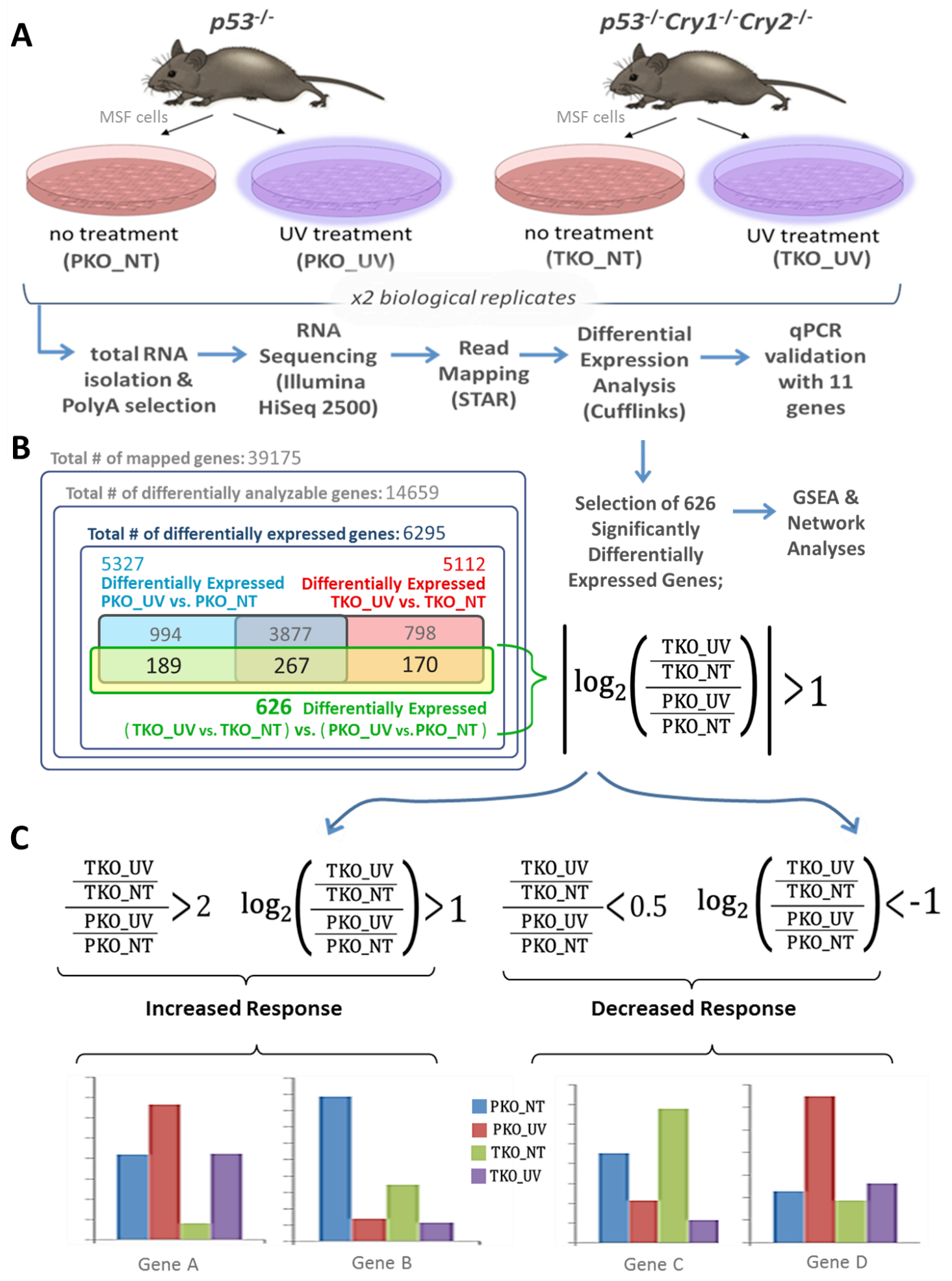

Fig. 1 
A

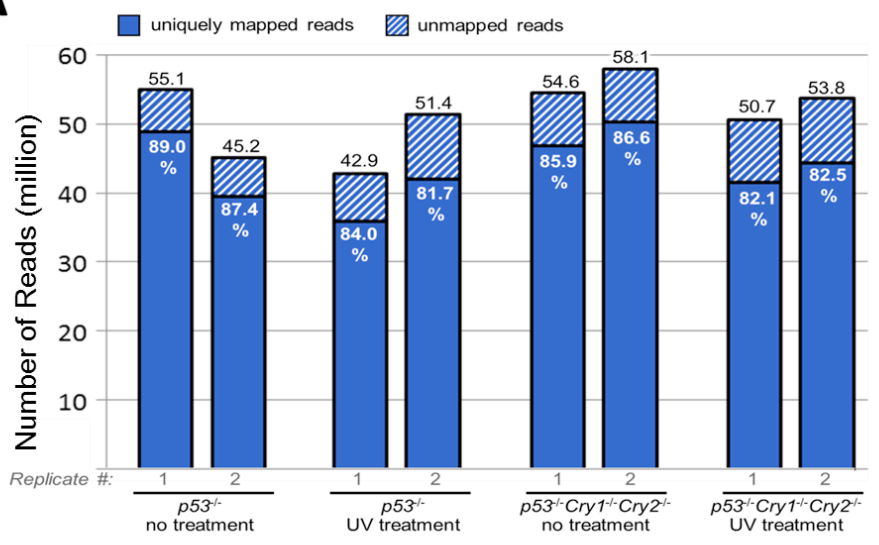

C

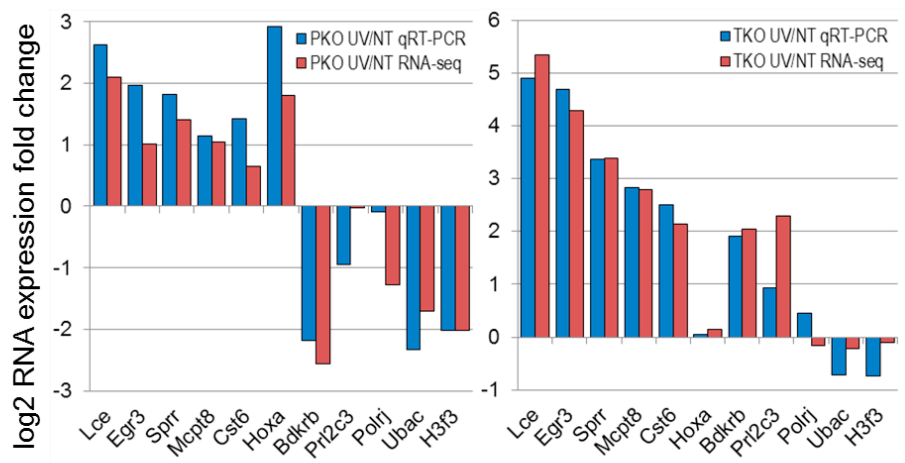

D 14659 differentially analyzable genes

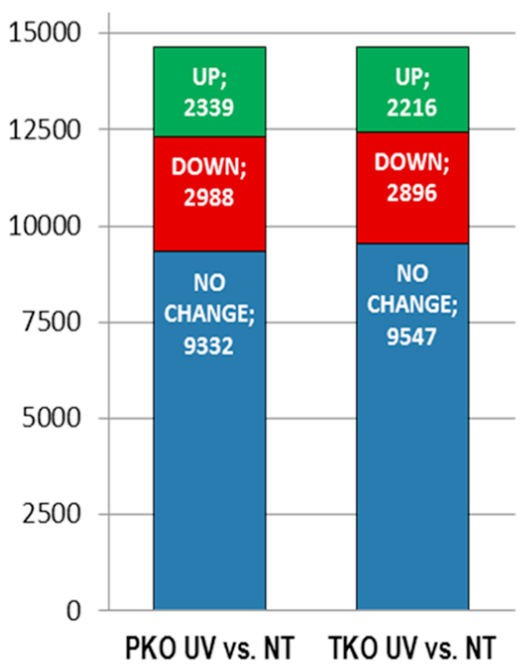

E

26 Significant DEGs

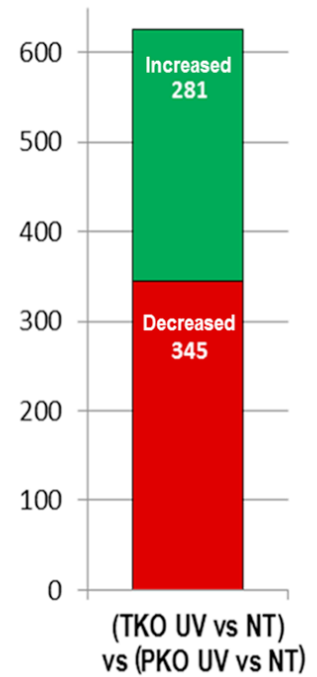

B
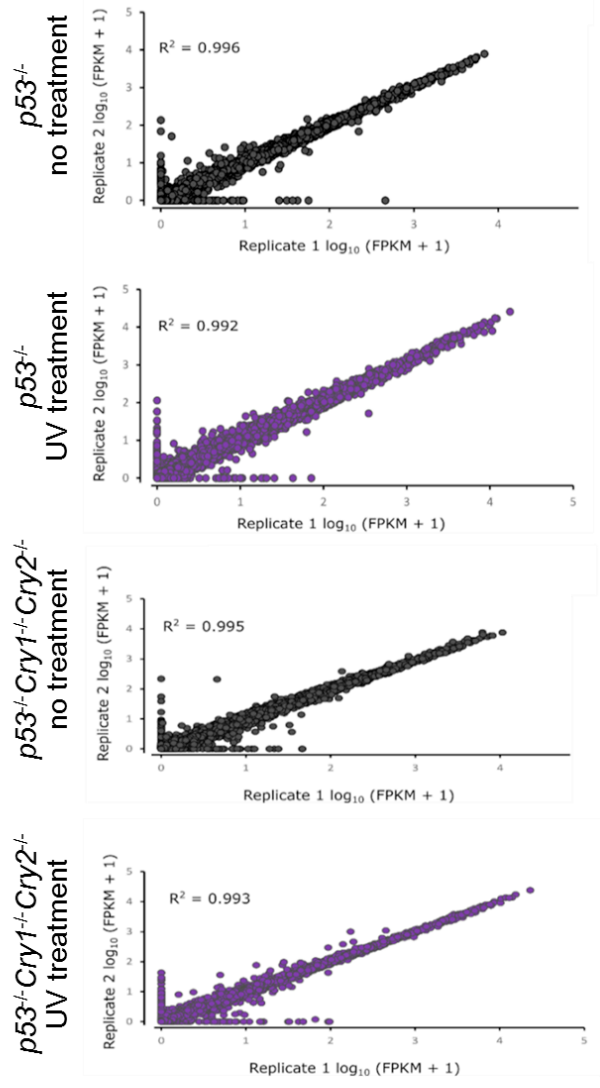

F $\quad 626$ Significant DEGs

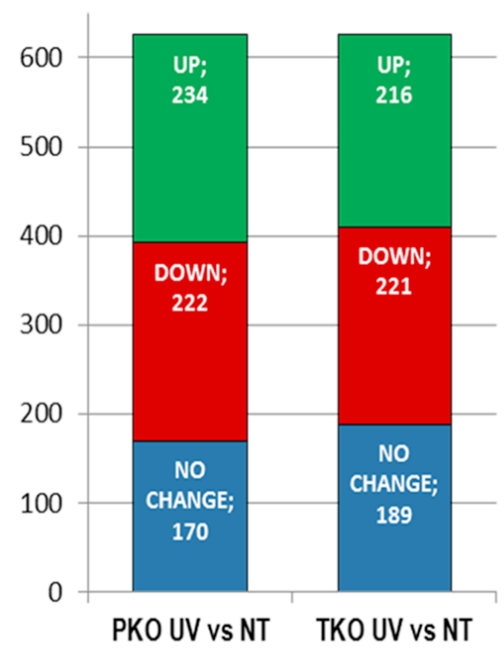

Fig. 2 


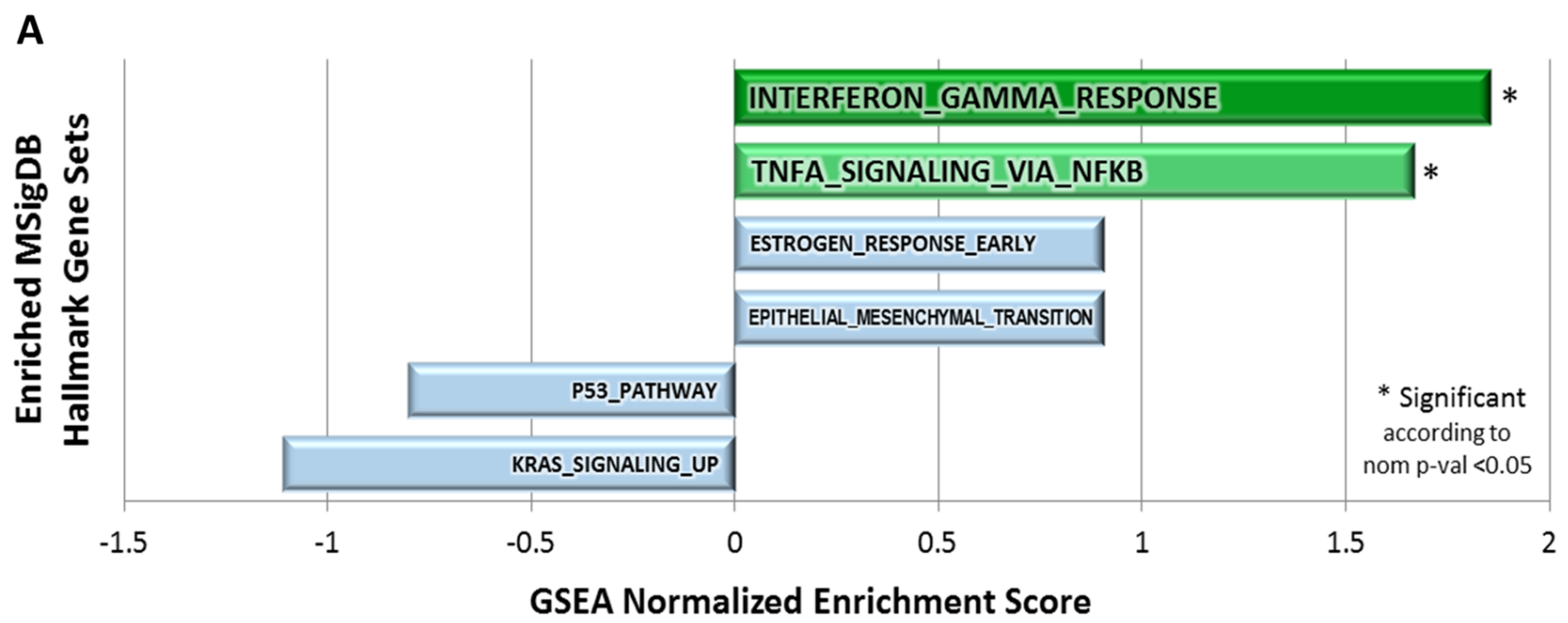

B GSEA Interferon-y response

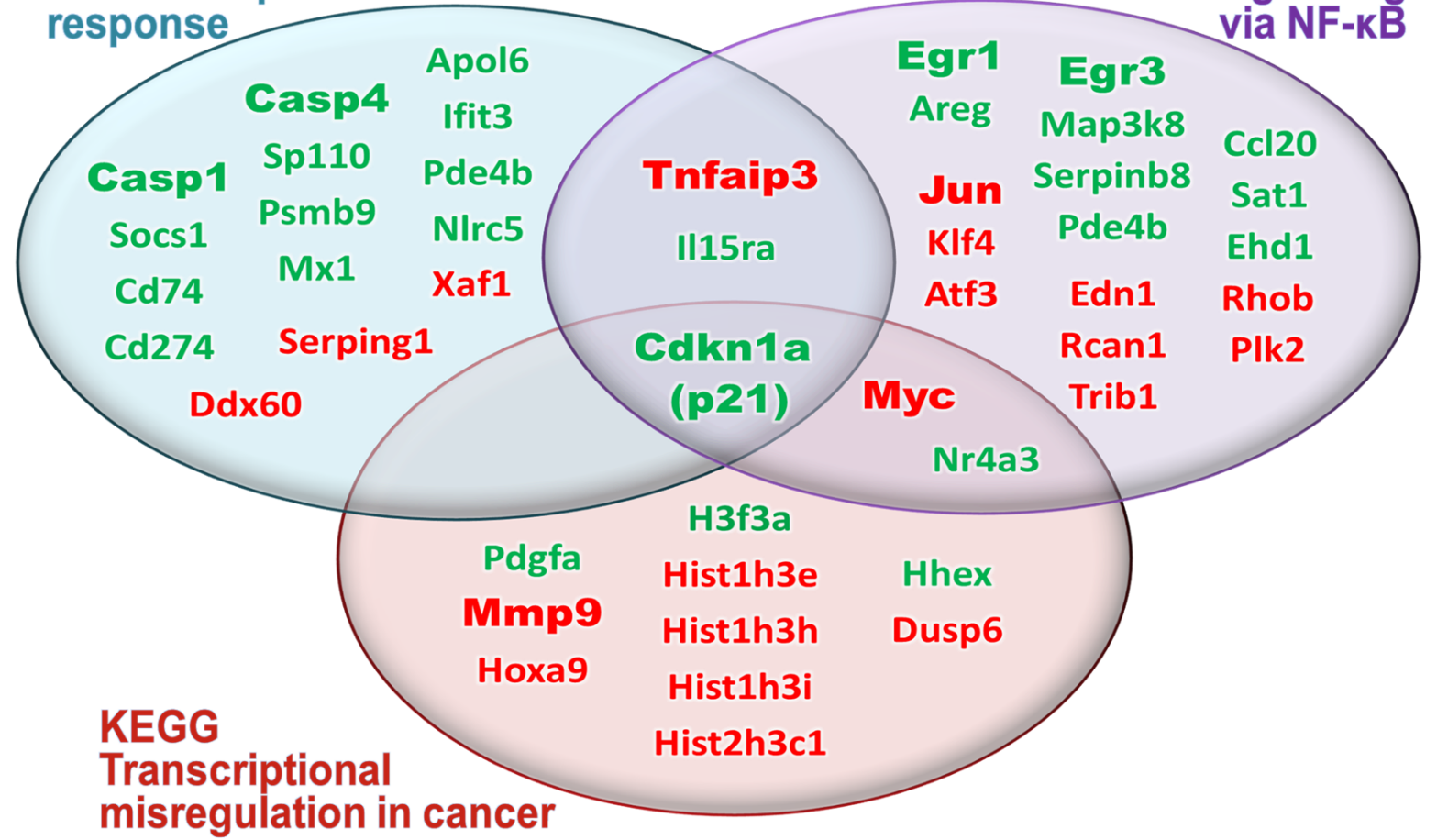

Fig. 3 


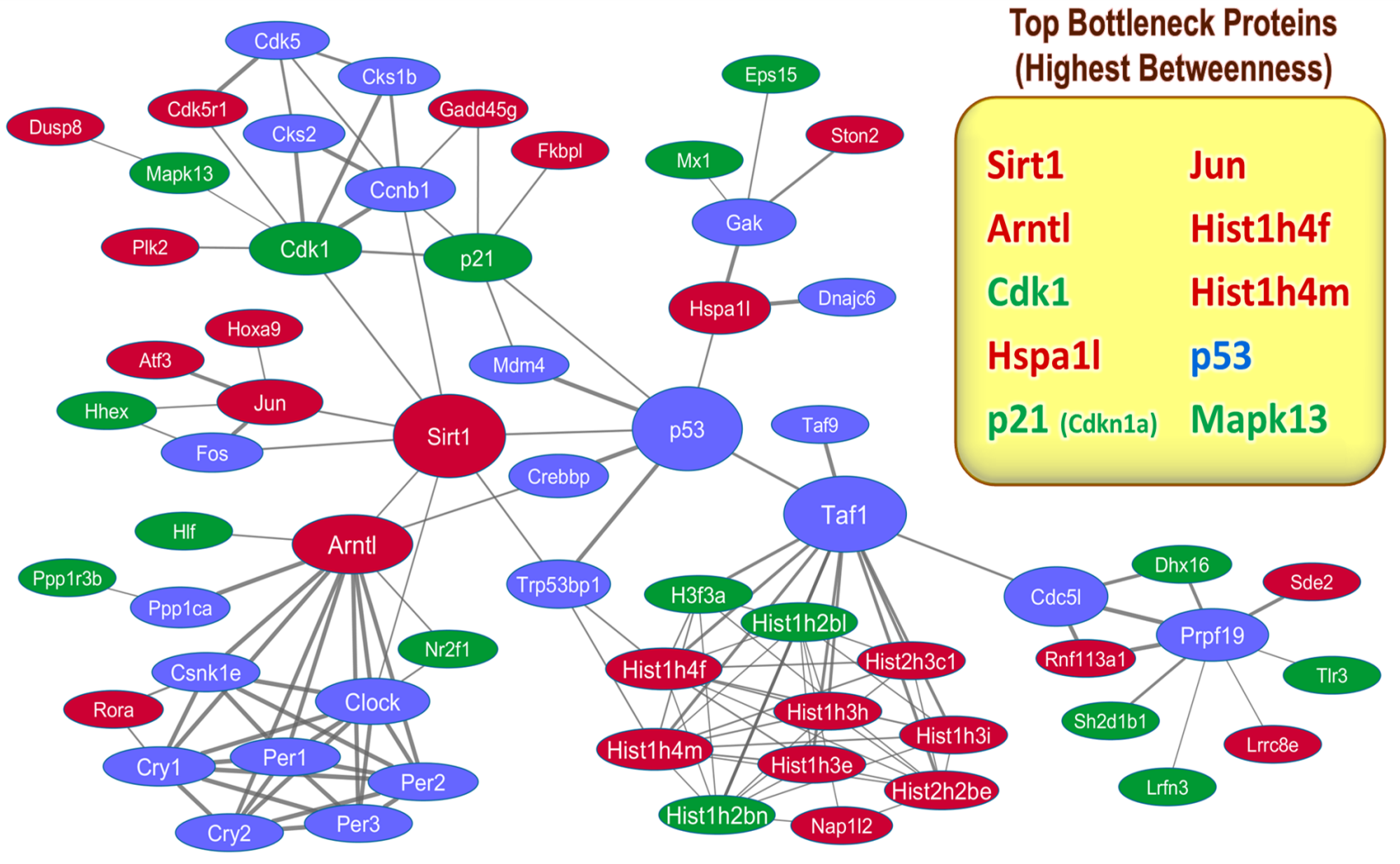

Fig. 4

23 
A

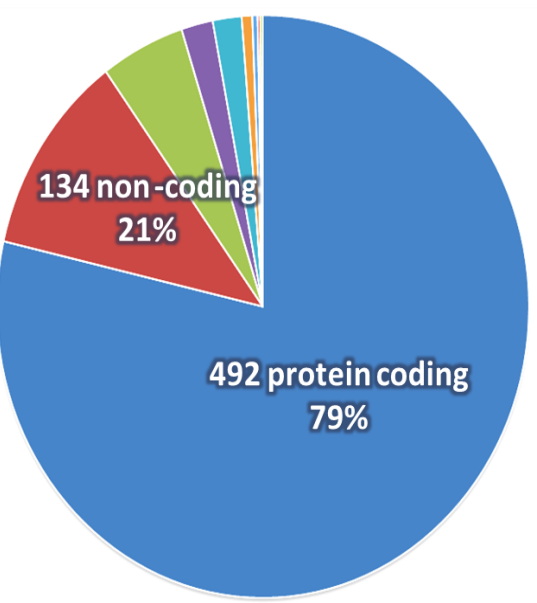

626 significant DEGs
B

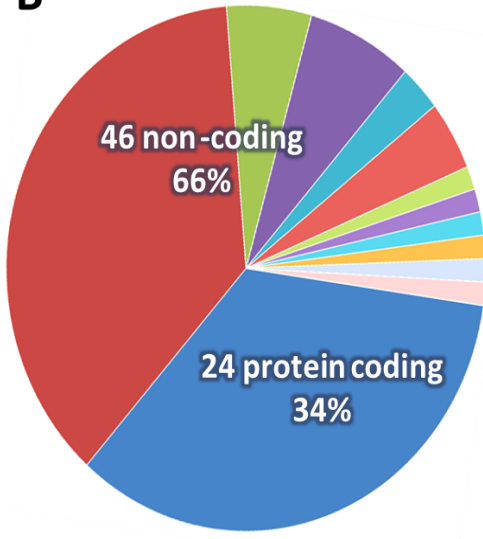

70 significant DEGs eliminated by zero errors $\square$ protein coding gene

$\square$ pseudogene

$\square$ lincRNA gene

$\square$ unclassified non-coding RNA gene

$\square$ antisense IncRNA gene

$\square$ unclassified gene

$\square$ polymorphic pseudogene

$\square$ snRNA gene

$\square$ bidirectional promoter IncRNA gene

$\square$ gene segment

$\square$ miRNA gene

$\square$ scRNA gene

$\square$ snoRNA gene

$\square$ SRP RNA gene

Fig. 5 


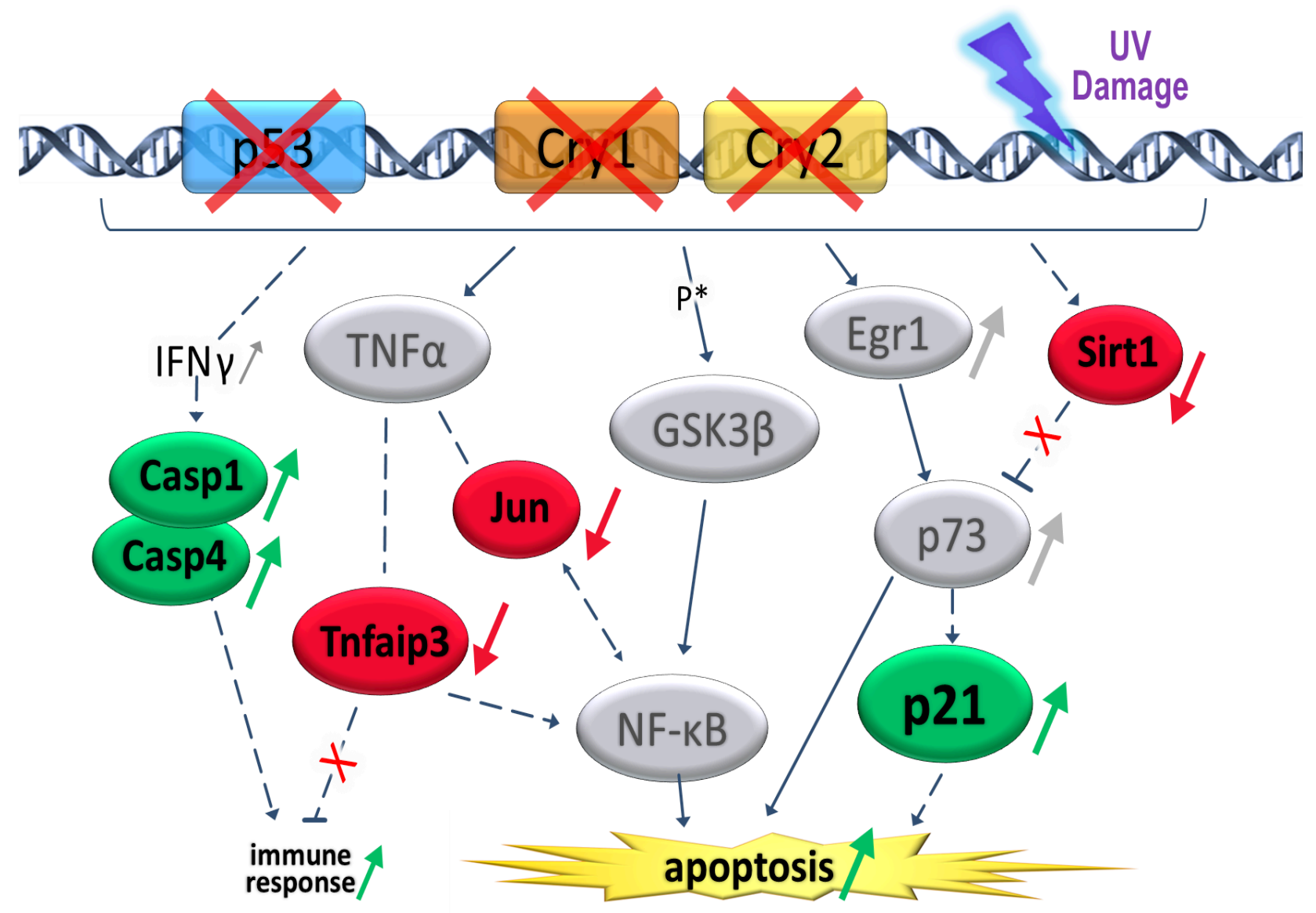

Fig. 6 